\title{
Wheat germ cell-free system-based production of hemagglutinin-neuraminidase glycoprotein of human parainfluenza virus type 3 for generation and characterization of monoclonal antibody
}

\author{
Satoko Matsunaga ${ }^{1}$, Shiho Kawakami ${ }^{1}$, Izumi Matsuo' , Akiko Okayama ${ }^{2}$, Hiroyuki Tsukagoshi ${ }^{3}$, \\ Ayumi Kudoh ${ }^{7}$, Yuki Matsushima ${ }^{4}$, Hideaki Shimizu ${ }^{4}, N_{0 b}$ hiko Okabe ${ }^{4}$, Hisashi Hirano ${ }^{2}$, \\ Naoki Yamamoto ${ }^{5}$, Hirokazu Kimura ${ }^{6}$ and Akihide Ryo ${ }^{1}$ *
}

${ }^{1}$ Department of Microbiology, Yokohama City University School of Medicine, Kanagawa, Japan

2 Proteome Analysis Center, Yokohama City University School of Medicine, Kanagawa, Japan

${ }^{3}$ Gunma Prefectural Institute of Public Health and Environmental Sciences, Gunma, Japan

${ }^{4}$ Kawasaki City Health and Safety Research Center, Kanagawa, Japan

${ }^{5}$ Department of Microbiology, Yong Loo Lin School of Medicine, National University of Singapore, Singapore

${ }^{6}$ Infectious Disease Surveillance Center, National Institute of Infectious Diseases, Tokyo, Japan

\section{Edited by:}

Yasuko Tsunetsugu Yokota, National Institute of Infectious Diseases, Japan

\section{Reviewed by:}

Yoshimichi Okayama, Nihon University

School of Medicine, Japan

Masatsugu Obuchi, Toyama Institute

of Health, Japan

Takeshi Saraya, Kyorin University

School of Medicine, Japan

${ }^{*}$ Correspondence:

Akihide Ryo, Department of Microbiology, Yokohama City University School of Medicine, 3-9 Fuku-ura, Kanazawa-ku, Yokohama, Kanagawa 236-0004, Japan e-mail: aryo@yokohama-cu.ac.jp
Human parainfluenza virus 3 (HPIV3) commonly causes respiratory disorders in infants and young children. Monoclonal antibodies (MAbs) have been produced to several components of HPIV3 and commercially available. However, the utility of these antibodies for several immunological and proteomic assays for understanding the nature of HPIV3 infection remain to be characterized. Herein, we report the development and characterization of MAbs against hemagglutinin-neuraminidase $(\mathrm{HN})$ of HPIV3. A recombinant full-length HPIV3-HN was successfully synthesized using the wheat-germ cell-free protein production system. After immunization and cell fusion, 36 mouse hybridomas producing MAbs to HPIV3-HN were established. The MAbs obtained were fully characterized using ELISA, immunoblotting, and immunofluorescent analyses. Of the MAbs tested, single clone was found to be applicable in both flow cytometry and immunoprecipitation procedures. By utilizing the antibody, we identified HPIV3-HN binding host proteins via immunoprecipitation-based mass spectrometry analysis. The newly-developed MAbs could thus be a valuable tool for the study of HPIV3 infection as well as the several diagnostic tests of this virus.

Keywords: human parainfluenza virus 3 , monoclonal antibody, cell-free protein synthesis, proteomics

\section{INTRODUCTION}

Human parainfluenza viruses (HPIVs) are major causes of lower respiratory infections in infants, young children, the immunocompromised, the chronically ill, and the elderly (Glezen et al., 1984; Counihan et al., 2001; Weinberg et al., 2009). HPIVs belong to the Paramyxoviridae family of medium-sized enveloped viruses and their genomes are organized on a single negative-sense strand of RNA. Of the four predominant serotypes of HPIV, the HPIV3 is the most frequently detected in viral infections in respiratory tracts. In fact, HPIV3 is second only to respiratory syncytial virus (RSV) as a cause of pneumonia and bronchiolitis in infants and young children. The HPIV3 genome contains approximately 15,000 nucleotides encoding at least six common structural proteins (3'-N-P-C-M-F-HN-L-5'; Storey et al., 1984; Tashiro and Homma, 1985; Wechsler et al., 1985). The two envelope glycoproteins, hemagglutinin-neuraminidase $(\mathrm{HN})$ and fusion $(\mathrm{F})$, are necessary for viral entry, cell-fusion and syncytium formation (Horvath et al., 1992; Hu et al., 1992; Takimoto et al., 2002; Porotto et al., 2012).

The HN protein is found on the lipid envelope of HPIVs, where it likely exists as a tetramer. HN is important for HPIV3 infection of host cells because it functions in virus-host cell attachment via sialic acid receptors and in virus release from cells with its neuraminidase activity (Huberman et al., 1995; Porotto et al., 2001; Chu et al., 2013). HN can be recognized by the host immune system and antibodies against epitopes within $\mathrm{HN}$ can neutralize its activity through the inhibition of the function and/or activity of either hemagglutinin or neuraminidase. Therefore, characterization of the protein structure and function of $\mathrm{HN}$ is of great importance for the understanding of HPIV3 infection and the host immunity against this virus.

Polyclonal antibodies and monoclonal antibodies (MAbs) against HPIV3 antigens have previously been generated, and animal antiserum to HPIV3 HN is also commercially available. However, the antibodies demonstrated cross-reactivity to other HPIV family viruses and exhibited relatively high non-specific background staining in immunoassays (Goswami and Russell, 1983; Waner etal., 1985). MAbs have been also produced to several components of HPIV3 (van Wyke Coelingh et al., 1985; Rydbeck etal., 1986). Although these commercially available MAbs have been shown to be specific, the utility of these antibodies for several immunological and/or proteomic analyses for 
understanding the nature of HPIV3 infection have not been fully delineated.

In our current study, we utilized the innovative wheat germ cellfree protein production system to generate the antigen protein. The main advantage of the cell-free protein system is the synthesis of proteins that are properly folded and that possess biological activity because the proteins are expressed in a eukaryotic cell system. Moreover, this system is capable of producing toxic proteins, such as viral antigens, that cause severe cytotoxicity or interference with host cellular physiology. By utilizing HPIV3-HN protein synthesized by the wheat cell-free system, we established multiple hybridoma clones producing MAbs that specifically targeted the viral antigen and were applicable for several immunoassays. Furthermore, we used the MAbs in proteomic analyses for identifying host proteins that potentially act as $\mathrm{HN}$ binding partners.

\section{MATERIALS AND METHODS CONSTRUCTION OF WHEAT GERM CELL-FREE EXPRESSION VECTOR}

Amplification of HN fragment from HPIV3 (Strain C243) genome was performed with the following primers: forward (5'-AGGAGTAAAGTTACGCAATCCAA) and reverse (5' ATATTTCCCTTTTGTCTATTGTCTG). For producing the expression vector of wheat germ cell-free system, The HN openreading frame was amplified by PCR using the forward primer (5'-GAGAGGATCCCATGGAATACTGGAAGCAT) and reverse primer (5'-GAGAGCGGCCGCTTAACTGCAGCTTTTTGGA). The amplified fragment was subcloned using BamH I and Not I into pEU-His or pEU-bls-S1 (bls; biotin ligase site) vectors. Biotinylated HN mutants were generated using the PrimeSTAR Mutagenesis Basal kit (TakaraBio, Otsu, Japan) according to the manufacturer's instructions.

HN protein fragments comprising CT, TM, and stalk regions were generated by the wheat cell-free system based on the template cDNA amplified from clinical isolates of HPIV1 (GeneBank No. JQ901977; Beck et al., 2012), HPIV2 (GeneBank No. AF533010; Skiadopoulos et al., 2003), and Mumps (GeneBank No. AB699704; Momoki, 2013) using following primer sets. HPIV1: (5'-ATGCTTATACTCTGGAGTCAAGA) and (5'TCTAGCAAAACRTGAAGTTGAG); HPIV2: (5'-AAAAACCTAAAATAAGCACGAA); and (5'-CCATTCTGGCCTATATYATAAT), Mumps: (5'-TTACTTATAAGACTGCGGTGC) and (5'-CTTGCAATGAGTTCTACTCTGA). Synthetic cDNA encoding the CT, $\mathrm{TM}$ and stalk regions of Sendai virus (UniProtKB entry no. P04853; Miura et al., 1985) was generated by Operon Biotechnologies (Huntsville, WI, USA). The amplified fragments were subcloned into pEU-bls-S1 vectors using In-fusion cloning system (TakaraBio) according to the manufacturer's instructions.

\section{CELL-FREE PROTEIN SYNTHESIS AND PURIFICATION}

In vitro transcription and cell-free protein synthesis were performed as described (Takai and Endo, 2010; Takai et al., 2010). For cell-free protein synthesis, the ENDEXT Wheat Germ Expression S Kit (CellFree Sciences, Yokohama, Japan) was used according to the manufacturer's instructions for the bilayer translation method. GST fusion and Biotinylated proteins were produced as previously described (Sawasaki et al., 2008; Takahashi et al., 2012).
His-HPIV3-HN protein, used for the generation of hybridoma, was synthesized using the robotic synthesizer (Protemist XE; CellFree Sciences) according to the manufacturer's instructions. The cell-free translation reaction mixture $(15 \mathrm{ml})$ was separated into soluble and insoluble fractions by centrifugation at 15,000 rpm for $15 \mathrm{~min}$. The insoluble fraction was lysed by the addition of $8 \mathrm{M}$ Urea at room temperature for $6 \mathrm{~h}$, then mixed with $\mathrm{Ni}$ sepharose High Performance beads (GE Healthcare, Waukesha, WI, USA) in the presence of $20 \mathrm{mM}$ imidazole. The beads were washed three times with washing buffer $(20 \mathrm{mM}$ Tris- $\mathrm{HCl} \mathrm{pH}$ 7.5, $500 \mathrm{mM} \mathrm{NaCl}$ ) containing $40 \mathrm{mM}$ imidazole. His-HN proteins were then eluted with washing buffer containing $8 \mathrm{M}$ Urea, $500 \mathrm{mM}$ imidazole. Amicon Ultra centrifugal filters (Millipore, Bedford, MA, USA) were used to concentrate the purified His-HN proteins by approximately 10 - to 20 -fold. The protein concentration was determined using the Bradford method with bovine serum albumin (BSA) as a protein standard.

\section{IMMUNIZATIONS AND GENERATION OF HYBRIDOMAS}

Monoclonal antibodies specific for HPIV3-HN were generated using the previously described hybridoma technology (Kimura et al., 1994). In brief, $300 \mu \mathrm{g}$ of N-terminal, His-tagged full-length HPIV3-HN protein was injected into the footpad of Balb/c mice using keyhole limpet hemocyanin as an adjuvant. Four weeks later, spleen cells were isolated and fused to the myeloma cell line SP2/O using polyethylene glycol 1500 (PEG 1500) as previously described (Kimura et al., 1996). Isotype determination was performed with the mouse MAb isotyping test kit (Bio-Rad, Hercules, CA, USA) according to the manufacturer's protocol.

\section{ELISA}

Microtiter plates coated with HPIV3-HN were incubated with threefold serial dilutions of each antibody (starting from 1:300 dilution of a hybridoma culture supernatant). After incubation with a peroxidase-conjugated secondary antibody and washing with PBS, the colorimetric signal of tetramethylbenzidine was detected by measuring the absorbance at $405 \mathrm{~nm}$ (Abs) using a plate reader.

\section{IMMUNOBLOTTING}

Recombinant HPIV3-HN proteins (equivalent to $\sim 100 \mathrm{ng}$ ) or HPIV3-infected HeLa cell lysates were separated by 10\% SDS-Gel and transferred onto a PVDF membrane (Millipore). The membrane was then soaked in Tris-buffered saline (TBS) containing 5\% (w/v) skim milk for $1 \mathrm{~h}$ and incubated with a MAb (hybridoma supernatant, 1:10 dilution) in TBS containing $0.1 \%$ (v/v) Tween20 (TBST) overnight at $4^{\circ} \mathrm{C}$. After washing three times with TBST, the membrane was incubated for $1 \mathrm{~h}$ in TBST containing goat-anti mouse IgG-HRP antibody (1:10000; GE Healthcare, Buckinghamshire, UK). After washing three times in TBST, the blot was detected with ImmobilonWestern Chemiluminescent HRP Substrate (Millipore) using FluorChem FC2 (Alpha Innotech, Santa Clara, CA, USA) in accordance to the manufacturer's protocol.

\section{IMMUNOPRECIPITATION}

HeLa cells were infected with HPIV3 (Strain C243) at multiplicity of infection (MOI) of 100 for $48 \mathrm{~h}$. The cells were lysed with 
immunoprecipitation buffer (50 mM Tris- $\mathrm{HCl} \mathrm{pH} 7.5,100 \mathrm{mM}$ $\mathrm{NaCl}, 0.5 \%$ NP-40, $200 \mu \mathrm{M}$ PMSF, $50 \mu \mathrm{MVO}_{4}, 2 \mu \mathrm{g} / \mathrm{ml}$ Aprotinin, $5 \mu \mathrm{g} / \mathrm{ml}$ Leupeptin, $1 \mu \mathrm{g} / \mathrm{ml}$ Pepstatin A). For immunoprecipitation assay, cell lysate or wheat germ cell extract generating full-length HPIV3-HN was incubated with the individual MAb (hybridoma supernatant), preclearing overnight using protein $\mathrm{A} / \mathrm{G}$ sepharose beads, for $2 \mathrm{~h}$ at $4^{\circ} \mathrm{C}$. After washing three times with immunoprecipitation buffer, immunocomplexes were eluted from the beads with $2 x$ SDS sample buffer. Then, the bound protein was analyzed by immnoblotting.

\section{IMMUNOFLUORESCENCE}

HeLa cells were grown on coverslips for $24 \mathrm{~h}$ and the cells were infected with HPIV3 (MOI = 100); mock-infected cells served as the control. At $48 \mathrm{~h}$ post-infection, the cells were washed with PBS before fixation with 3\% formalin in PBS at room temperature for $15 \mathrm{~min}$. Cells were washed twice with PBS for $5 \mathrm{~min}$ followed by $100 \%$ methanol for $10 \mathrm{~min}$ at $-20^{\circ} \mathrm{C}$. Cells were permeabilized with PBS containing $0.05 \%$ Triton X-100 for $10 \mathrm{~min}$ at room temperature. The cells were incubated with individual primary antibodies for $1 \mathrm{~h}$ at room temperature. After being washed twice with PBS, the cells were incubated with secondary antibodies for $1 \mathrm{~h}$ at room temperature. The nucleus was counterstained with 4',6-diamidino-2-phenylindole (DAPI). Microscopic imaging was performed with an FV1000-D confocal laser scanning microscope (Olympus, Tokyo, Japan) equipped with a 60x oil-immersion objective.

\section{FLOW CYTOMETRY}

HeLa cells were seeded in 6-well plates at a concentration of $2 \times 10^{5}$ per well $24 \mathrm{~h}$ before infection, and the cells were washed with medium containing $0.0001 \%$ trypsin and infected with HPIV3 $(\mathrm{MOI}=100)$; mock-infected cells were used as a control. At 4 days post-infection, cells were harvested in PBS containing $5 \mathrm{mM}$ EDTA and washed twice with PBS. The cells were then incubated with either MAbs (hybridoma supernatant; 5X dilution) or non-immunized hybridoma supernatant in PBS containing 2\% Blocking One (NACALAI TESQUE, INC., Kyoto, Japan) for $1 \mathrm{~h}$ at room temperature. After being washed twice with PBS, the cells were incubated with phycoerythrin (PE)-conjugated anti-mouse IgG antibodies (Beckman Coulter, Fullerton, CA, USA) for $1 \mathrm{~h}$ at room temperature. Flow cytometric analysis of the cells $(10,000$ cells per sample) was performed on a FACScanto II instrument (BD Biosciences, San Jose, CA, USA).

\section{EPITOPE MAPPING AND SPECIFICITY OF MAbS USING ALPHASCREEN ASSAY}

The AlphaScreen assay was performed using 384-well ProxiPlates (PerkinElmer, Boston, MA, USA). Biotinylated virus proteins or GST (negative control) were incubated with a 40-fold dilution of MAbs (hybridoma supernatant) in $15 \mu \mathrm{l}$ of binding mixture containing reaction buffer $(100 \mathrm{mM}$ Tris- $\mathrm{HCl}, \mathrm{pH} 7.5$, $1 \mathrm{mg} / \mathrm{ml} \mathrm{BSA,} 0.01 \%$ Tween-20) at $26^{\circ} \mathrm{C}$ for $30 \mathrm{~min}$. Then, combine $10 \mu \mathrm{l}$ of the detection mixture containing $0.1 \mu \mathrm{l}$ protein G-conjugated acceptor beads and $0.1 \mu \mathrm{l}$ streptavidin-coated donor beads (AlphaScreen IgG detection kit, PerkinElmer) in reaction buffer were incubated at $26^{\circ} \mathrm{C}$ for $1 \mathrm{~h}$. Antigen-antibody interactions were analyzed using an Envision microplate reader (PerkinElmer).

\section{PROTEOMIC ANALYSIS}

Cell lysate from HPIV3-infected or mock-infected HeLa cells were immunoprecipitated with \#21 MAb. The binding proteins were separated by SDS-PAGE and transferred to PVDF membranes. For LC-MS/MS analysis, the membranes digested with trypsin. LC-MS/MS analysis was performed using a TripleTOF MS (TripleTOF 5600 system, AB SCIEX, Foster City, CA, USA) and the Analyst version 1.6 TF (AB SCIEX) coupled to an DiNaAP (KYA Technologies, Tokyo, Japan). Prior to injection into the mass spectrometer, the tryptic digests were filtered through a Ultrafree-MC, GV $0.22 \mu \mathrm{m}$ filter (Millipore), then loaded onto a reverse phase pre-column (HiQ sil C18W-3, $500 \mu \mathrm{m}$ id $\times 1 \mathrm{~mm}$, KYA Technologies) and resolved on a nanoscale HiQ sil C18W$3(100 \mu \mathrm{m}$ id $\times 10 \mathrm{~cm}$; KYA Technologies $)$ at a flow rate of $200 \mathrm{~nL} / \mathrm{min}$ with a gradient of acetonitrile/0.1\% (v/v) formic acid. Peptides were separated using a 30 min gradient from 5 to $100 \%$ solvent B $[0.1 \%(\mathrm{v} / \mathrm{v})$ formic acid $/ 80 \%(\mathrm{v} / \mathrm{v})$ acetonitrile]. Solvent A was $0.1 \%(\mathrm{v} / \mathrm{v})$ formic acid/2\% (v/v) acetonitrile. The obtained MS and tandem-MS data were searched against the human protein sequences in the Swiss-Prot database (version Jan 2013, 20233sequences) using the Protein Pilot software 4.5 (AB SCIEX).

\section{RESULTS}

\section{PRODUCTION OF MAbs}

In our current study, we attempted to produce the full-length HPIV3-HN by the wheat cell-free production system (Figure 1A). Complementary DNA encoding HPIV3-HN open-reading frame was sub-cloned into pEU-His, the expression vector designed specifically for the wheat germ cell-free system for expressing His-tagged protein. Consequently, His-tagged HPIV3-HN protein was synthesized by this procedure in a large scale. Since HPIV3-HN exhibited high insolubility in regular buffer, the protein was suspended in the buffer including $8 \mathrm{M}$ urea. This suspended His-tagged HPIV3-HN was further purified using Nisepharose followed by the elusion with imidazole. Balb/c mice were then immunized with purified full-length HPIV3-HN protein. After 4 weeks, splenocytes were isolated and hybridomas were created (Figure 1A). Finally, 36 stable hybridomas were obtained and designated \#1 to \#36. The resulting hybridomas were screened by ELISA with plates coated with HPIV3-HN protein conjugated with BSA. Of the 36 hybridoma clones established, seven clones $(\# 4, \# 5, \# 7, \# 10, \# 14, \# 21, \# 23)$ exhibited relatively high absorbances (Figure 1B). Titration analyses with diluted hybridoma supernatants or antigenic HN protein revealed that these seven antibodies had higher specificities and intensities than control hybridoma supernatants (Figure 1C). These seven hybridomas were processed for further characterization.

\section{IMMUNOBLOTTING ANALYSES OF MAbS}

We next tested the MAbs in immunoblotting analysis. First, recombinant HPIV3-HN protein was separated by SDS-PAGE followed by immunoblotting with MAbs isolated from the seven 


\section{A}

pEU-E01-His $_{\text {TEV }}$-HPIV3-HN
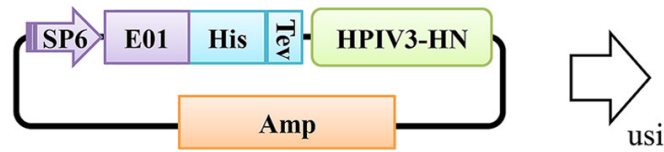

In vitro transcription

\&

translation

using wheat germ cell-free system

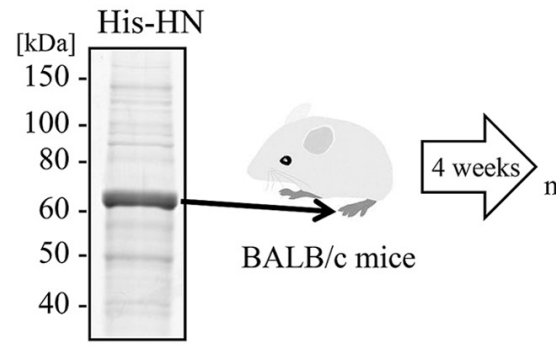

Fusion with mouse myeloma cells

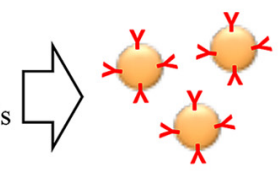

36 hybridoma cells

B

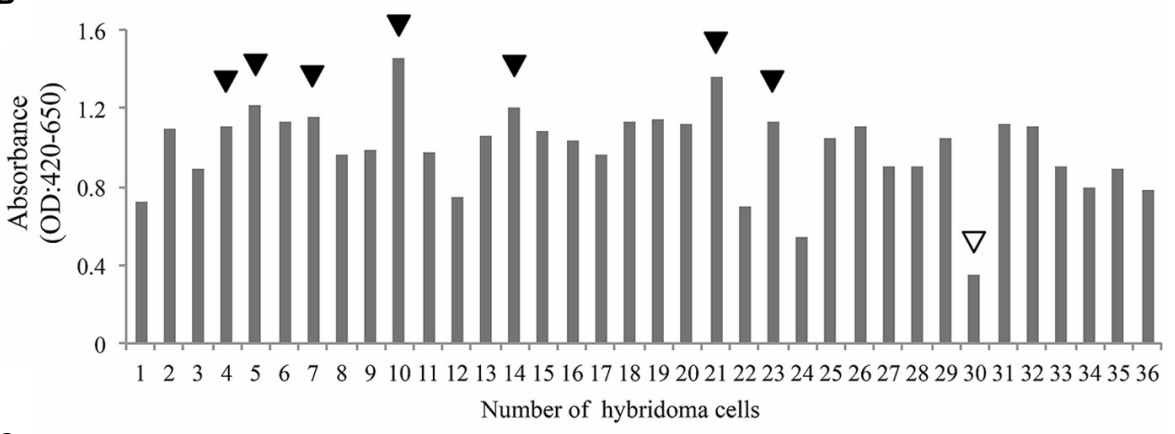

C

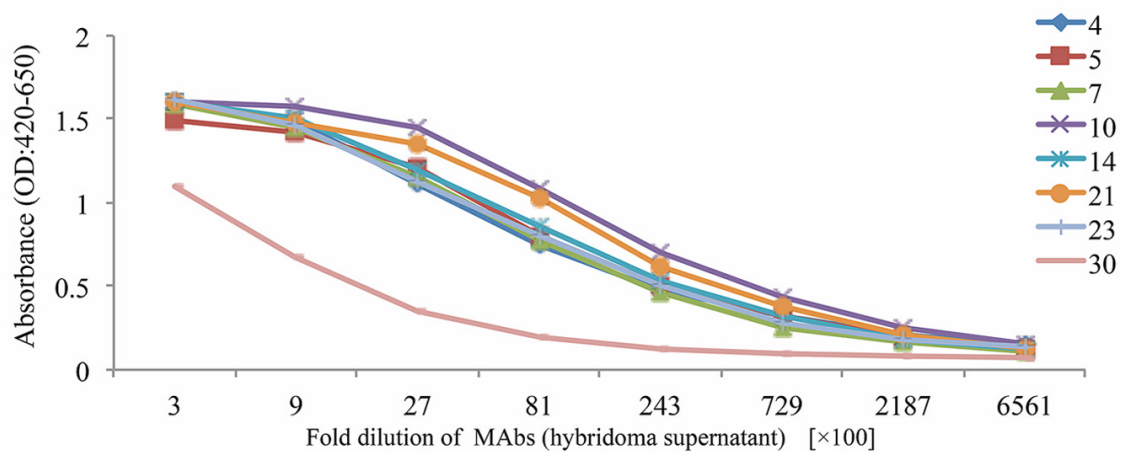

FIGURE 1 | Production of hybridoma cells generating anti-HPIV3-HN antibodies. (A) Schematic diagram of hybridoma cells production generating anti-HPIV3-HN monoclonal antibody (MAb). The recombinant Histidine-tagged recombinant HPIV3-HN (His-HN) protein was produced by wheat germ cell-free system and then purified by nickel-chelated sepharose beads in the presence of $8 \mathrm{M}$ urea. The purified protein was separated by SDS-PAGE and visualized by CBB-staining. Purified His-HN protein was injected into the footpad of Balb/c mice. After 4 weeks, immunized mouse splenocytes were fused with myeloma cells and then 36 hybridoma cells were established. SP6, SP6 promoter sequence; E01, translation enhancer sequence; His, Histidine-tagged sequence; TEV; TEV protease recognized sequence. (B,C) The specificity of MAbs (hybridoma supernatant) evaluated by ELISA. The specificity of 36 MAbs in 2700-fold dilution was determined (B). The black arrows indicate the selected MAbs while the white arrow depicts a selected clone as a negative control (clone no. \#30). The selected eight MAbs were diluted at serial points and analyzed by ELISA (C). hybridomas. As shown in Figure 2A, all seven MAbs recognized a single band that corresponded to the recombinant protein. Next, immunoblotting analysis was performed with cell lysates from HeLa cells either infected- or mock-infected with HPIV3. The all seven MAbs detected a $63 \mathrm{kDa}$ protein band that was consistent with the molecular mass of the HN protein (Figure 2B). No other bands were detected by the MAbs indicating that they specifically recognized $\mathrm{HN}$.

\section{IMMUNOFLUORESCENT ANALYSIS}

We next performed an immunofluorescent (IF) analysis of HPIV3infected HeLa cells with seven MAbs (Figure 2C). Three of the MAbs (\#7, \#21, and \#23) exhibited prominent IF staining of the infected cells, while the other four MAbs had no detectable IF staining. The IF staining with the antibodies revealed that $\mathrm{HN}$ protein exhibited granular staining pattern throughout cytoplasm and plasma membrane in HPIV3-infected cells (Figure 2D), which 
A

Recombinant HN

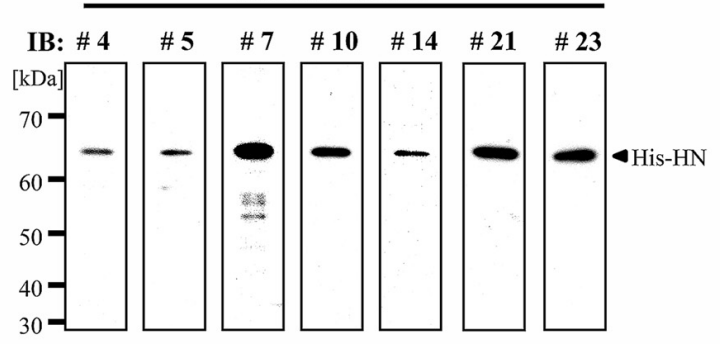

B

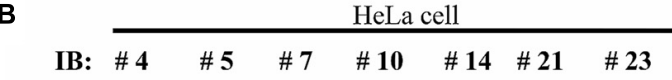
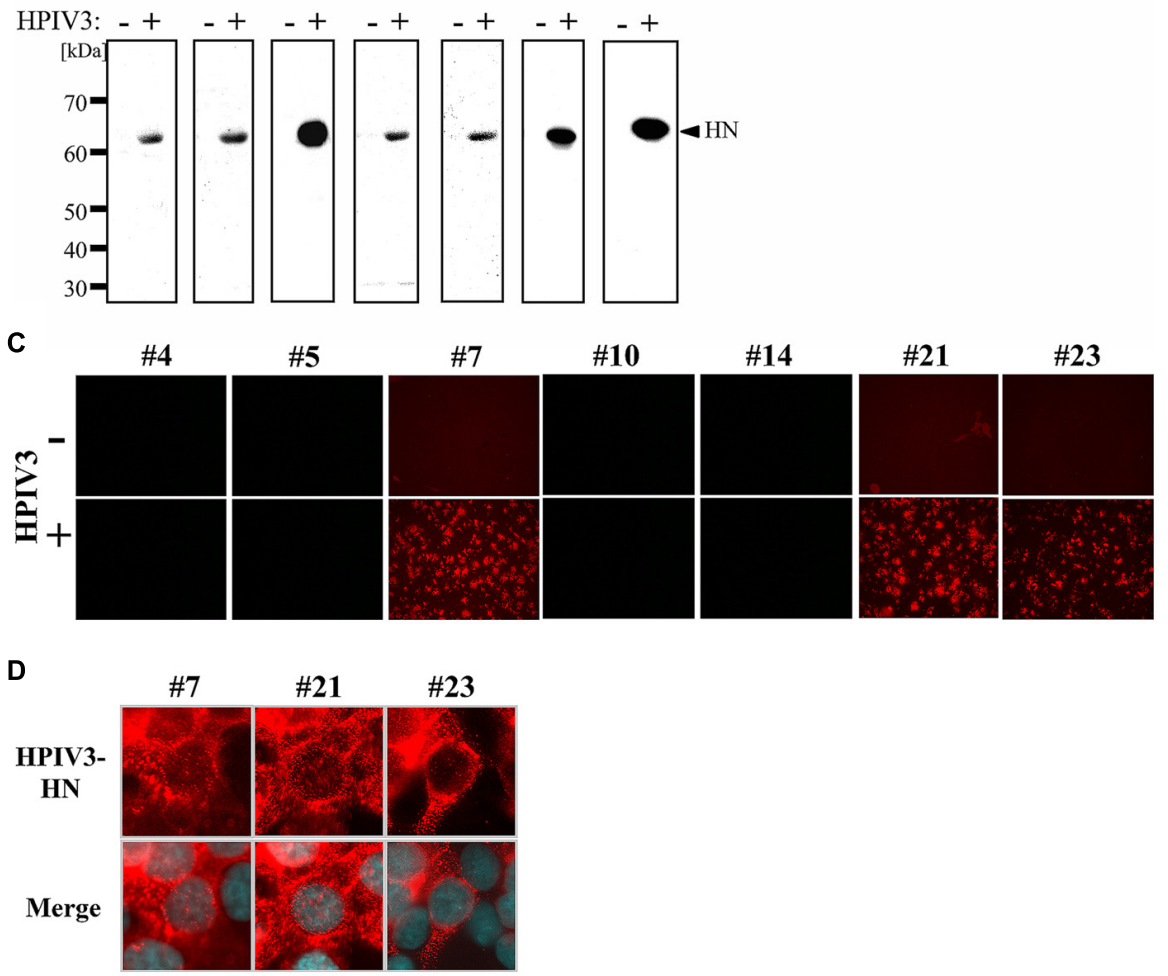

FIGURE 2 | Immunoblotting and immunofluorescent analysis.

(A,B) Detection sensitivity of the MAbs for recombinant His-HN (A) or HPIV3-infected cell lysate (B). Recombinant HPIV3-HN (100 ng) was separated using $12.5 \%$ SDS-gel and transferred to a PVDF membrane, followed by incubation with MAbs (hybridoma supernatants) at a 1:10 dilution (A). HeLa cells were infected or mock-infected with HPIV3. After $48 \mathrm{~h}$, cells were lysed with SDS-PAGE loading buffer. The total protein was separated in $12.5 \%$ SDS-gel and immunobloted with indicated MAbs (B). (C,D) Immunofluoresent analysis of $H N$ (red) in HPIV3-infected HeLa cells. HeLa cells were infected or mock-infected with HPIV3. After $48 \mathrm{~h}$, cells were fixed, and then stained with MAbs (hybridoma supernatant; red) and DAPI (blue). Confocal microscopic analysis was performed at $40 \times$ (C) and at $600 \times$ magnifications (D) was consistent with previous studies (Ali and Nayak, 2000; Stone and Takimoto, 2013). The control mock-infected cells did not show any signals when stained with the antibodies (Figure 2C). We thus selected the three MAbs (\#7, \#21, and \#23) for further characterization.

\section{IMMUNOPRECIPITATION}

We next examined whether these selected antibodies were useful in immunoprecipitation analysis. The wheat germ extract containing full-length HPIV3-HN with glutathione-S-transferase (GST) tag was incubated with protein $\mathrm{A} / \mathrm{G}$-coated sepharose beads (GE Helthcare) together with the three selected antibodies or non-immunized mouse IgG antibody. The precipitated samples were subjected to immunoblotting analysis with anti-GST antibody. The GST tagged HN protein was precipitated by all selected antibody (\#7, \#21, and \#23) but not non-immunized IgG (Figure 3A).

\section{FLOW CYTOMETRY ANALYSIS}

We next addressed the usability of the antibodies \#7, \#21, and \#23 in flow cytometry analysis. HeLa cells infected with HPIV3 were stained with the antibodies and then subjected to FACS analysis. 


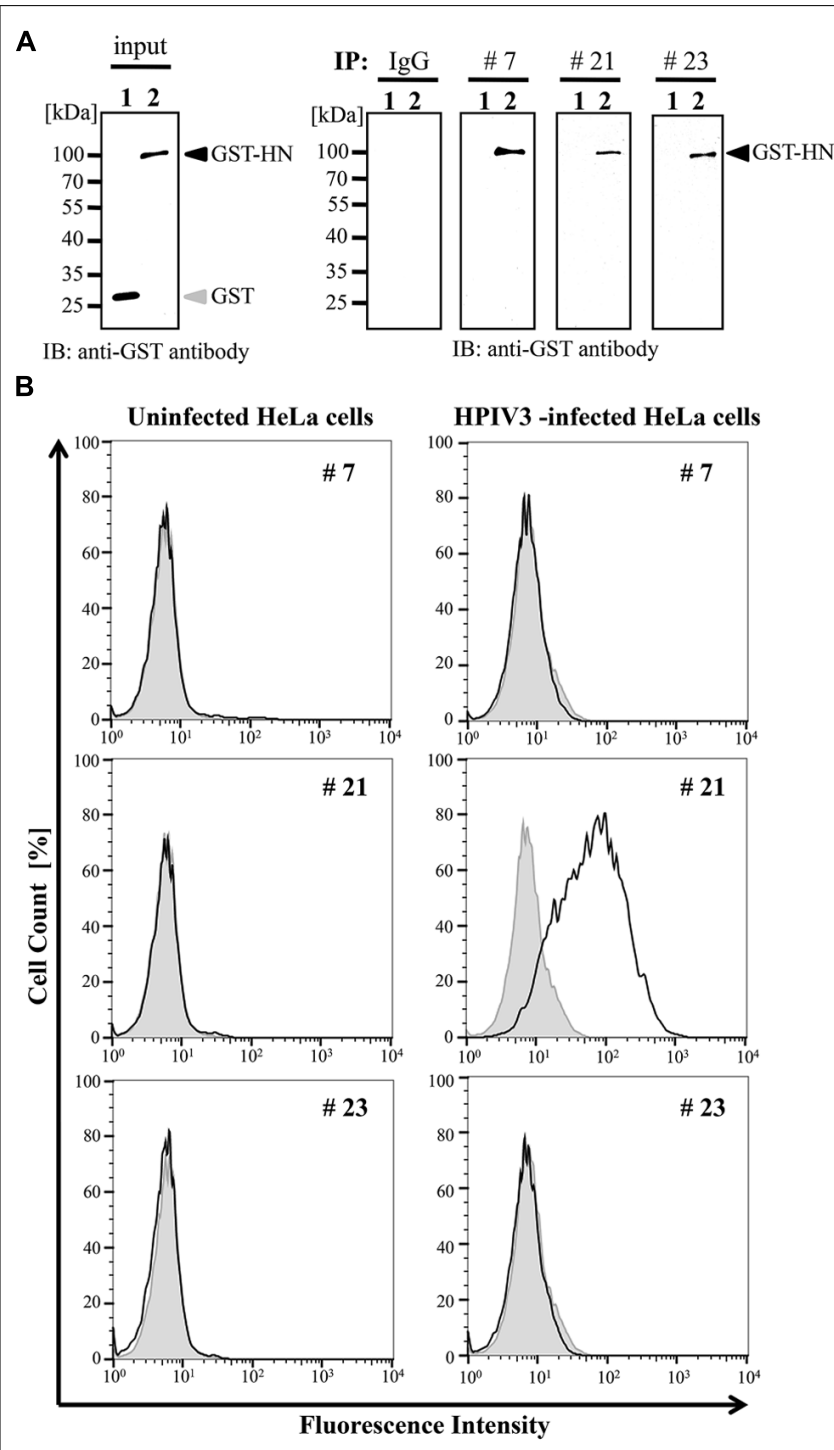

FIGURE 3 | Immunoprecipitation and flow cytometry assay. (A) Recombinant GST-HPIV3-HN or GST protein was immnoprecipitated with either \#7, \#21, \#23 MAbs, or lgG (negative control), respectively. Then bound proteins were analyzed with immunoblotting using anti-GST antibody. (B) HPIV3-infected or uninfected HeLa cells were harvested at 4 days post-infection, followed by incubation with indicated MAbs. The cells were then fixed and stained with anti-mouse secondary antibody. The population of stained cells was calculated by flow cytometry. The shaded histogram shows negative hybridoma supernatant and the bold line shows specific MAbs.

Our results demonstrated that only \#21 MAbs could specifically detect virus-infected cell populations, and distinguished between viral-infected and uninfected cell population with flow cytometory (Figure 3B).

\section{EPITOPE MAPPING OF MAbs}

To determine the binding domain of the MAbs within the HPIV3-HN protein, we synthesized six different deletion mutants of HPIV3-HN as depicted in Figure 4A. All of the deletion mutants contained $\mathrm{N}$-terminal biotin tag were incubated with the antibodies (\#7, \#21, or \#23), followed by the addition of AlphaScreen streptavidin donor and protein A acceptor beads, as depicted in Figure 4B. The reactivity was measured and calculated by the level of the AlphaScreen luminescent signal. The results showed that two MAbs (\#7 and \#23) reacted against the cytoplasmic tail (CT) of HPIV3-HN whereas \#21 MAb detected extracellular stalk region of the protein (Figures 4C-E). This result is fully consistent with the result of flow cytometry analysis (Figure 3B).

\section{SPECIFICITY OF MAb}

We next investigate the specificity of MAbs using $\mathrm{HN}$ protein frangments derived from HPIV1, Sendai virus (SeV), HPIV2, and Mumpus virus $(\mathrm{MuV})$. Partial $\mathrm{HN}$ protein fragments containing cytoplasmic tail (CT), transmembrane domain (TM), and stalk region were produced with a N-terminal biotin tag by wheat cellfree system, and then incubated with the antibodies (\#7, \#21, or \#23) followed by the AlphaScreen (Figure 5A). The antigen reactivity was measured based on the level of the AlphaScreen luminescent signal (Figure 5B). Notably, there was no crossreactivity to $\mathrm{HN}$ proteins derived from other Paramyxoviruses except for HPIV3. This was also confirmed by immunoblotting analysis (Figure 5C). These results indicate the specificity of the antibodies for HPIV3-HN.

\section{PROTEOMIC ANALYSIS}

We next utilized our newly developed \#21 MAb for the identification of host proteins that bind to HPIV3-HN during HPIV3 infection. Cell lysate from HPIV3-infected or mockinfected cells were immunoprecipitated with \#21 MAb. Precipitated samples were collected and then digested with trypsin followed by LC-MS/MS analysis (Figure 6A). Annotation analysis using the Swiss-Prot database revealed that 10 proteins were putative $\mathrm{HN}$ binding proteins (Figure 6B). Based on the number of corrected peptides, top four proteins (HSP70, HSP90, tubulin, alpha 1c, and SERPINA3) were selected as the most likely candidates for association with $\mathrm{HN}$ and subjected to further binding analysis. The pull-down analysis of the host proteins with recombinant HPIV3-HN was performed. The subsequent immunoblotting analysis demonstrated that HPIV3-HN could indeed interact with these four proteins (Figure 6C). These results demonstrate the availability of our newly-developed antibody in comprehensive proteomic analysis.

\section{DISCUSSION}

Herein we produced HPIV3-HN proteins by the wheat cell-free system, and created MAbs that selectively target the HPIV3-HN protein. Characterization of the most potent MAb confirmed the antigen-specificity and usability in various applications including immunoblotting, immnofluorescent, flow cytometry, and immunoprecipitation analyses. Furthermore, the MAb could capture the endogenous HN protein from HPIV3-infected cells to identify HN-binding host proteins via mass spectrometry-based proteomic analysis. Our current results demonstrated the generation of useful antibody against HPIV3-HN and also shed new light 


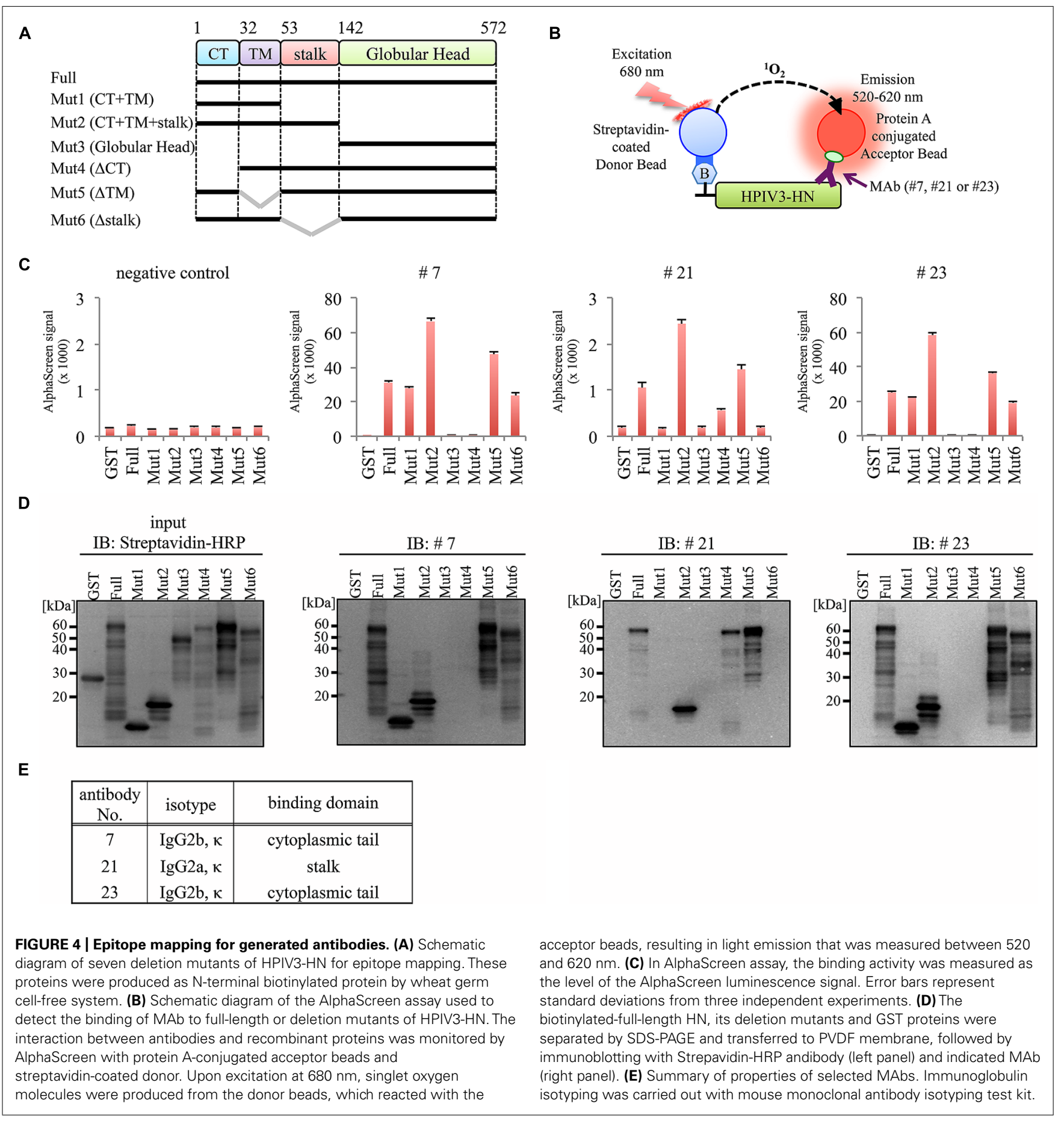

on the unexplored molecular link between the PIV3-HN and host proteins.

Currently, cell-based production (e.g., E. coli system or baculovirus-insect cell system) of recombinant virus proteins has been widely used. However, it is often difficult to produce sufficient quantities of viral antigens in the conventional cell-based system because many viral antigens are usually insoluble, cytotoxic, and are expressed in the inclusion body fraction. In contrast, the cell-fee protein production system enables the

synthesis of toxic proteins that are otherwise excluded from production in live cells. Among the cell-free approaches, the wheat germ cell-free system employs a eukaryotic translation system that warrants the synthesis of properly folded and biologically active proteins similar to proteins that are expressed in living mammalian cells (Endo and Sawasaki, 2005, 2006). These advantages underscore the suitability and availability of the wheat germ cell-free system for the generation of antigenic proteins that can be used for animal immunizations to generate 


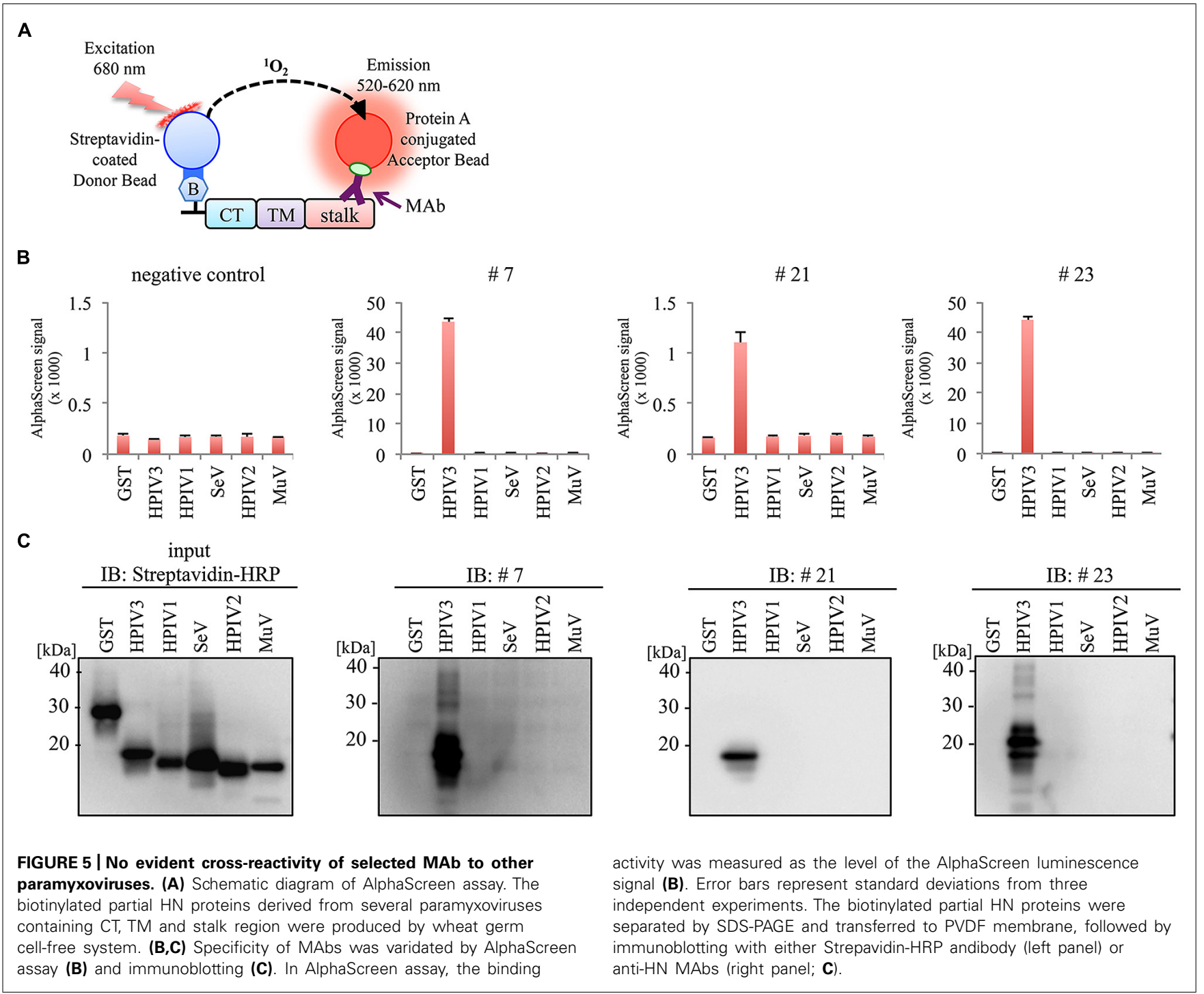

MAbs. Our current study clearly demonstrated the benefit of using viral proteins synthesized by the wheat germ cell-free system to efficiently produce the MAbs against the viral antigen. Using this approach, we have created MAbs against HPIV3-HN that detected both denatured and native forms of the antigen. These MAbs were useful in various immunological assays including ELISA, IF, immunoblotting, and immunoprecipitation. Further careful studies of structural aspects are needed to determine whether the MAb can affect the virus infectivity.

We identified Hsp70 as a putative HN binding protein. Several previous studies demonstrated that Hsp70 was involved in the regulation of other RNA viruses. Hsp70 is known to associate with viral $\mathrm{PB} 1$ and $\mathrm{PB} 2$ subunits of influenza A virus, and it negatively regulated the expression of viral proteins in infected cells (Li etal., 2011). In another study, Hantavirus infection induced the expression of HSP70 that interacted with nucleocapsid protein and its overexpression suppressed viral infection in Vero E6 cells (Yu et al., 2009). In contrast, Hsp70 was found to positively regulate rabies viral infection. Indeed, rabies infection induced the cellular expression of Hsp70 and accumulation in Negri body-like structures, which are the site of viral transcription and replication. Inhibition of Hsp70 resulted in a significant decrease of viral mRNAs, viral proteins, and virus particles (Lahaye et al., 2009, 2012). Taken together these results indicated a pivotal role of Hsp70 in viral replication and the pathogenicity of viral infection. Hsp70 binds and regulates many cellular proteins, as well as viral proteins (Pratt and Toft, 2003; Mayer, 2005; Silver and Noble, 2012), and the effects of Hsp70 on viral infection are diverse and unique between different viral species or cell systems. Further studies are required to investigate the precise molecular mechanism by which the association of Hsp70 and HN proteins mediate HPIV3 replication.

We also found that PIV3-HN can interact with Serpin3a. Serpin3a, as also known as alpha-1-antichymotrypsin, is a member of serpin proteins involved in the inhibition of serine and other types of proteases (Bauman et al., 2002). In humans, the majority 
A

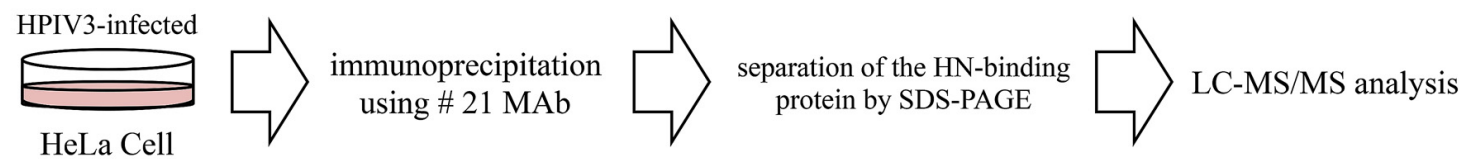

B

\begin{tabular}{|c|c|c|c|c|c|}
\hline $\begin{array}{c}\text { Accession } \\
\text { number }\end{array}$ & name & peptide & Da & Molecular function & Subcellular localization \\
\hline \hline P08107 & HSP70 & 10 & 70,052 & Chaperone & cytoplasm \\
\hline P08238 & HSP90 & 6 & 83,264 & Chaperone & cytoplasm \\
\hline Q9BQE3 & Tubulin alpha-1C & 6 & 49,895 & structural constituent of cytoskeleton & cytoplasm, cytoskeleton, microtubule \\
\hline P01011 & SERPINA3 & 6 & 47,651 & protease inhibitor & cytoplasm \\
\hline P07195 & LDH-B & 5 & 36,638 & oxidoreductase & mitochondrion \\
\hline P04406 & GAPDH & 5 & 36,053 & oxidoreductase, transferase & cytoplasm, cytoskeleton, membrane, nucleus \\
\hline P10809 & HSP60 & 4 & 61,055 & chaperone & cell membrane, cytoplasm, membrane, nucleus \\
\hline P06733 & MBP-1 & 4 & 47,169 & lyase, repressor & cytoplsdm, nucleus \\
\hline P14618 & PKM & 4 & 57,937 & kinase, transferase & cytoplasm \\
\hline P60842 & eIF4A1 & 3 & 46,154 & helicase, hydrolase, initiation factor & \\
\hline
\end{tabular}

C

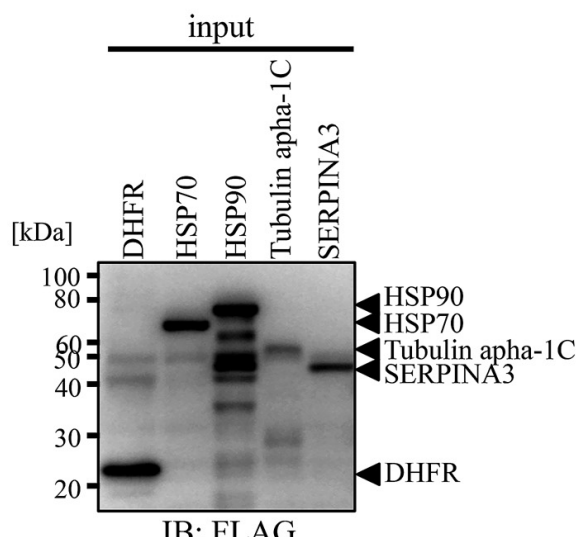

IB: FLAG

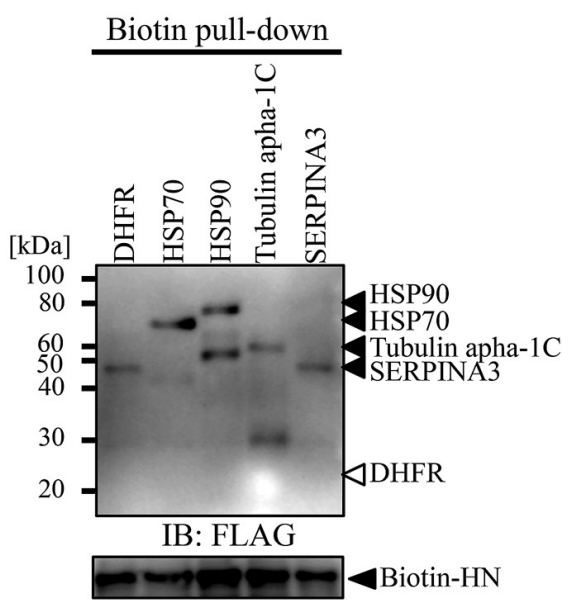

(C) FLAG-tagged HSP70, HSP90, tubulin alpha 1C, or SERPINA3 proteins were mixed with HPIV3-HN. Samples were pull-down with the streptavidin magnetic beads and the collected proteins were separated by SDS-PAGE. The bound protein detected by immunoblotting analysis with anti-FLAG antibody. The right arrows indicated the position of each protein. of serpins regulates the functions of proteases involved in the response against body's injuries such as coagulation, fibrinolysis, inflammation, wound healing and tissue repair. Serpins have been also implicated in various pathologies in respiratory system such as airway hyperresponsiveness (AHR) and asthma (Sivaprasad etal., 2011). The physical interaction of HPIV3HN with serpin3a may be involved in hindering the function of Serpin3a toward respiratory disorders. Further analysis may shed new light on understanding the etiology of HPIV3-induced asthma.

In summary, we utilized the wheat cell-free production system to create and characterize MAbs that may be useful in various immunological applications. Our newly-developed MAbs could thus provide a valuable means to explore HPIV3 infection in human cells.

\section{ACKNOWLEDGMENTS}

We thank Tomoe Suzuki for technical assistance, and Dr. Kei Miyakawa for FACS analysis. This work was partially supported by the fund for Creation of Innovation Centers for Advanced Interdisciplinary Research Areas Program in the Project for Developing Innovation Systems from the Ministry of Education, Culture, Sports, Science and Technology, and Research Grants from The Ministry of Health Labour and Welfare of Japan to Akihide Ryo.

\section{REFERENCES}

Ali, A., and Nayak, D. P. (2000). Assembly of Sendai virus: M protein interacts with $\mathrm{F}$ and $\mathrm{HN}$ proteins and with the cytoplasmic tail and transmembrane domain of F protein. Virology 276, 289-303. doi: 10.1006/viro.2000.0556

Bauman, S. J., Whinna, H. C., and Church, F. C. (2002). Serpins (serine protease inhibitors). Curr. Protoc. Protein Sci. Chap. 21, Unit 21.7. doi: 10.1002/0471140864.ps2107s26 
Beck, E. T., He, J., Nelson, M. I., Bose, M. E., Fan, J., Kumar, S., et al. (2012). Genome sequencing and phylogenetic analysis of 39 human parainfluenza virus type 1 strains isolated from 1997-2010. PLOS ONE 7:e46048. doi: 10.1371/journal.pone.0046048

Chu, F. L., Wen, H. L., Hou, G. H., Lin, B., Zhang, W. Q., Song, Y. Y., et al. (2013). Role of N-linked glycosylation of the human parainfluenza virus type 3 hemagglutinin-neuraminidase protein. Virus Res. 174, 137-147. doi: 10.1016/j.virusres.2013.03.012

Counihan, M. E., Shay, D. K., Holman, R. C., Lowther, S. A., and Anderson, L. J. (2001). Human parainfluenza virus-associated hospitalizations among children less than five years of age in the United States. Pediatr. Infect. Dis. J. 20, 646-653. doi: 10.1097/00006454-200107000-00003

Endo, Y., and Sawasaki, T. (2005). Advances in genome-wide protein expression using the wheat germ cell-free system. Methods Mol. Biol. 310, 145-167. doi: 10.1007/978-1-59259-948-6_11

Endo, Y., and Sawasaki, T. (2006). Cell-free expression systems for eukaryotic protein production. Curr. Opin. Biotechnol. 17, 373-380. doi 10.1016/j.copbio.2006.06.009

Glezen, W. P., Frank, A. L., Taber, L. H., and Kasel, J. A. (1984). Parainfluenza virus type 3: seasonality and risk of infection and reinfection in young children. J. Infect. Dis. 150, 851-857. doi: 10.1093/infdis/150. 6.851

Goswami, K. K., and Russell, W. C. (1983). Monoclonal antibodies against human paramyxovirus type 3 and against SV5 virus: preparation and preliminary characterization. J. Gen. Virol. 64, 1663-1672. doi: 10.1099/0022-1317-648-1663

Horvath, C. M., Paterson, R. G., Shaughnessy, M. A., Wood, R., and Lamb, R. A. (1992). Biological activity of paramyxovirus fusion proteins: factors influencing formation of syncytia. J. Virol. 66, 4564-4569.

Hu, X. L., Ray, R., and Compans, R. W. (1992). Functional interactions between the fusion protein and hemagglutinin-neuraminidase of human parainfluenza viruses. J. Virol. 66, 1528-1534.

Huberman, K., Peluso, R. W., and Moscona, A. (1995). Hemagglutininneuraminidase of human parainfluenza 3: role of the neuraminidase in the viral life cycle. Virology 214, 294-300. doi: 10.1006/viro.19 95.9925

Kimura, K., Nozaki, N., Enomoto, T., Tanaka, M., and Kikuchi, A. (1996). Analysis of M phase-specific phosphorylation of DNA topoisomerase II. J. Biol. Chem. 271, 21439-21445. doi: 10.1074/jbc.271.35.21439

Kimura, K., Nozaki, N., Saijo, M., Kikuchi, A., Ui, M., and Enomoto, T. (1994). Identification of the nature of modification that causes the shift of DNA topoisomerase II beta to apparent higher molecular weight forms in the M phase. J. Biol. Chem. 269, 24523-24526.

Lahaye, X., Vidy, A., Fouquet, B., and Blondel, D. (2012). Hsp70 protein positively regulates rabies virus infection. J. Virol. 86, 4743-4751. doi: 10.1128/JVI.06 501-11

Lahaye, X., Vidy, A., Pomier, C., Obiang, L., Harper, F., Gaudin, Y., et al. (2009). Functional characterization of Negri bodies (NBs) in rabies virus-infected cells: evidence that NBs are sites of viral transcription and replication. J. Virol. 83, 7948-7958. doi: 10.1128/JVI.00554-09

Li, G., Zhang, J., Tong, X., Liu, W., and Ye, X. (2011). Heat shock protein 70 inhibits the activity of Influenza A virus ribonucleoprotein and blocks the replication of virus in vitro and in vivo. PLOS ONE 6:e16546. doi: 10.1371/journal.pone. 0016546

Mayer, M. P. (2005). Recruitment of Hsp70 chaperones: a crucial part of viral survival strategies. Rev. Physiol. Biochem. Pharmacol. 153, 1-46. doi: 10.1007/s10254-004-0025-5

Miura, N., Nakatani, Y., Ishiura, M., Uchida, T., and Okada, Y. (1985). Molecular cloning of a full-length cDNA encoding the hemagglutinin-neuraminidase glycoprotein of Sendai virus. FEBS Lett. 188, 112-116. doi: 10.1016/00145793(85)80885-1

Momoki, T. S. (2013). Genotyping of mumps virus detected in Yokohama City from 1999 to 2010. Jpn. J. Infect. Dis. 66, 226-231. doi: 10.7883/yoken. 66.226

Porotto, M., Greengard, O., Poltoratskaia, N., Horga, M. A., and Moscona, A. (2001). Human parainfluenza virus type $3 \mathrm{HN}$-receptor interaction: effect of 4-guanidino-Neu5Ac2en on a neuraminidase-deficient variant. J. Virol. 75, 74817488. doi: 10.1128/JVI.75.16.7481-7488.2001
Porotto, M., Palmer, S. G., Palermo, L. M., and Moscona, A. (2012). Mechanism of fusion triggering by human parainfluenza virus type III: communication between viral glycoproteins during entry. J. Biol. Chem. 287, 778-793. doi: 10.1074/jbc.M111.298059

Pratt, W. B., and Toft, D. O. (2003). Regulation of signaling protein function and trafficking by the hsp90/hsp70-based chaperone machinery. Exp. Biol. Med. (Maywood) 228, 111-133.

Rydbeck, R., Orvell, C., Love, A., and Norrby, E. (1986). Characterization of four parainfluenza virus type 3 proteins by use of monoclonal antibodies. J. Gen. Virol. 67, 1531-1542. doi: 10.1099/0022-1317-678-1531

Sawasaki, T., Kamura, N., Matsunaga, S., Saeki, M., Tsuchimochi, M., Morishita, R., etal. (2008). Arabidopsis HY5 protein functions as a DNAbinding tag for purification and functional immobilization of proteins on agarose/DNA microplate. FEBS Lett. 582, 221-228. doi: 10.1016/j.febslet.2007. 12.004

Silver, J. T., and Noble, E. G. (2012). Regulation of survival gene hsp70. Cell Stress Chaperones 17, 1-9. doi: 10.1007/s12192-011-0290-6

Sivaprasad, U., Askew, D. J., Ericksen, M. B., Gibson, A. M., Stier, M. T., Brandt, E. B., et al. (2011). A nonredundant role for mouse Serpinb3a in the induction of mucus production in asthma. J. Allergy Clin. Immunol. 127, 254-261. doi: 10.1016/j.jaci.2010.10.009

Skiadopoulos, M. H., Vogel, L., Riggs, J. M., Surman, S. R., Collins, P. L., and Murphy, B. R. (2003). The genome length of human parainfluenza virus type 2 follows the rule of six, and recombinant viruses recovered from non-polyhexameric-length antigenomic cDNAs contain a biased distribution of correcting mutations. J. Virol. 77, 270-279. doi: 10.1128/JVI.77.1.270279.2003

Stone, R., and Takimoto, T. (2013). Critical role of the fusion protein cytoplasmic tail sequence in parainfluenza virus assembly. PLOS ONE 8:e61281. doi: 10.1371/journal.pone.0061281

Storey, D. G., Dimock, K., and Kang, C. Y. (1984). Structural characterization of virion proteins and genomic RNA of human parainfluenza virus 3. J. Virol. 52, 761-766.

Takahashi, H., Takahashi, C., Moreland, N. J., Chang, Y. T., Sawasaki, T., Ryo, A., et al. (2012). Establishment of a robust dengue virus NS3-NS5 binding assay for identification of protein-protein interaction inhibitors. Antiviral Res. 96, 305-314. doi: 10.1016/j.antiviral.2012.09.023

Takai, K., and Endo, Y. (2010). The cell-free protein synthesis system from wheat germ. Methods Mol. Biol. 607, 23-30. doi: 10.1007/978-1-60327-331-2_3

Takai, K., Sawasaki, T., and Endo, Y. (2010). The wheat-germ cell-free expression system. Curr. Pharm. Biotechnol. 11, 272-278. doi: 10.2174/138920110791 111933

Takimoto, T., Taylor, G. L., Connaris, H. C., Crennell, S. J., and Portner, A. (2002). Role of the hemagglutinin-neuraminidase protein in the mechanism of paramyxovirus-cell membrane fusion. J. Virol. 76, 13028-13033. doi: 10.1128/JVI.76.24.13028-13033.2002

Tashiro, M., and Homma, M. (1985). Protection of mice from wild-type Sendai virus infection by a trypsin-resistant mutant, TR-2. J. Virol. 53, 228-234.

van Wyke Coelingh, K. L., Winter, C., and Murphy, B. R. (1985). Antigenic variation in the hemagglutinin-neuraminidase protein of human parainfluenza type 3 virus. Virology 143, 569-582. doi: 10.1016/0042-6822(85) 90395-2

Waner, J. L., Whitehurst, N. J., Downs, T., and Graves, D. G. (1985). Production of monoclonal antibodies against parainfluenza 3 virus and their use in diagnosis by immunofluorescence. J. Clin. Microbiol. 22, 535-538.

Wechsler, S. L., Lambert, D. M., Galinski, M. S., Heineke, B. E., and Pons, M. W. (1985). Human parainfluenza virus 3: purification and characterization of subviral components, viral proteins and viral RNA. Virus Res. 3, 339-351. doi: 10.1016/0168-1702(85)90434-4

Weinberg, G. A., Hall, C. B., Iwane, M. K., Poehling, K. A., Edwards, K. M., Griffin, M. R., et al. (2009). Parainfluenza virus infection of young children: estimates of the population-based burden of hospitalization. J. Pediatr. 154, 694-699. doi: 10.1016/j.jpeds.2008.11.034

Yu, L., Ye, L., Zhao, R., Liu, Y.F., and Yang, S.J. (2009). HSP70 induced by Hantavirus infection interacts with viral nucleocapsid protein and its overexpression suppresses virus infection in Vero E6 cells. Am. J. Transl. Res. 1, 367-380. 
Conflict of Interest Statement: The authors declare that the research was conducted in the absence of any commercial or financial relationships that could be construed as a potential conflict of interest.

Received: 24 February 2014; accepted: 18 April 2014; published online: 13 May 2014. Citation: Matsunaga S, Kawakami S, Matsuo I, Okayama A, Tsukagoshi H, kudoh A, Matsushima Y, Shimizu H, Okabe N, Hirano H, Yamamoto N, Kimura H and Ryo A (2014) Wheat germ cell-free system-based production of hemagglutinin-neuraminidase glycoprotein of human parainfluenza virus type 3 for generation and characterization of monoclonal antibody. Front. Microbiol. 5:208. doi: 10.3389/fmicb.2014.00208
This article was submitted to Virology, a section of the journal Frontiers in Microbiology.

Copyright (C) 2014 Matsunaga, Kawakami, Matsuo, Okayama, Tsukagoshi, kudoh, Matsushima, Shimizu, Okabe, Hirano, Yamamoto, Kimura and Ryo. This is an open-access article distributed under the terms of the Creative Commons Attribution License (CC BY). The use, distribution or reproduction in other forums is permitted, provided the original author(s) or licensor are credited and that the original publication in this journal is cited, in accordance with accepted academic practice. No use, distribution or reproduction is permitted which does not comply with these terms. 